\title{
E-beam-Deposited Tungsten Contacts for Carbon Nanofiber Interconnect Test Devices.
}

\author{
N. Kanzaki, ${ }^{*}$ S. Maeda, ${ }^{*}$ P. Wilhite, ${ }^{*}$ T. Yamada, ${ }^{*}$ T. Saito, ${ }^{* *}$ and C. Y. Yang* \\ * Center for Nanostructures, Santa Clara University, Santa Clara, CA 95053 \\ ** Hitachi High-Technologies Corporation, Ibaraki, Japan
}

As the downward scaling of integrated circuits continues, the problem of electromigration in copper interconnects becomes increasingly severe. Carbon nanofiber (CNF) can potentially be a replacement for $\mathrm{Cu}$ in on-chip interconnects because of its excellent thermal and electrical properties and vertically aligned growth at low temperature. To consider CNF as an on-chip interconnect, the contact resistance between the $\mathrm{CNF}$ and the metal electrode must be optimized. Using lithography technique to form contact between $\mathrm{CNF}$ and metal requires a complicated and costly device fabrication process [1]. Thus we proposed an alternative method using focused ion beam (FIB) [2]. The resulting total resistance decreased by a factor of about 1000 from that for the pre-W-deposited device [2]. However, FIB has the risk of damaging the on-chip devices because of its high beam energy, and gallium from the ion beam could result in device contamination. To address this problem, we have developed a technique for $\mathrm{W}$ deposition using a well-controlled electron beam in a variablepressure scanning electron microscope (VP-SEM). In this technique, the source gas is delivered via a specially designed gas injection system (GIS) [3] and guided by the focused electron beam to yield deposition on a selected target at a lower energy than FIB.

Fig. 1 shows a schematic of the GIS, which is installed in the chamber of a VP-SEM. The source gas for tungsten is $\mathrm{WF}_{6}$. In this study, the working distance inside the SEM chamber is set to $25 \mathrm{~mm}$, and the distance between the device surface and the nozzle tip is within $0.1 \mathrm{~mm}$. The electron beam acceleration voltage is $30 \mathrm{kV}$ with a beam current of $150 \mu \mathrm{A}$. The target area is exposed to the ebeam for $30 \mathrm{~min}$. Fig. 2a shows the SEM image of W-CNF-Au electrode contact formed. The size of the $\mathrm{W}$ spot is in the 300-500 $\mathrm{nm}$ range. In this study, we measured the resistances of seven devices before and after $\mathrm{W}$ deposition and the resistances of all devices decrease from $\mathrm{M} \Omega$ to $\mathrm{k} \Omega$ as a result of the deposited W contacts (Fig. 2b). Further, as shown in Fig. 2c for four of these devices, the resistance is not affected by current stressing. These behaviors are similar to those observed for devices with FIB-deposited W contacts [2].

We also prepared additional test devices for two-point (2PP) and four-point probe (4PP) measurements to yield the contact resistance between $\mathrm{CNF}$ and $\mathrm{W}$ and the resistivity of CNF. The test device after $\mathrm{W}$ deposition is shown in Fig. 3a. Both 2PP and 4PP measured results show linear- $I$ $V$-behaviors (Fig. 3b). The resistance of CNF test device between Au2 and Au3 obtained using 2PP measurement is $4.39 \mathrm{k} \Omega$. On the other hand, the $4 \mathrm{PP}$ result measured with a voltmeter across Au2 and $\mathrm{Au} 3$ is $3.90 \mathrm{k} \Omega$. Therefore, the total contact resistance between $\mathrm{CNF}$ and $\mathrm{W}$, and between the measurement probes and Au contact pads, is $0.49 \mathrm{k} \Omega$. The resulting extracted CNF resistivity is 3.82 $\times 10^{-3} \Omega \mathrm{cm}$. These values are similar to those obtained for CNF test devices with Au/Ti contacts fabricated using e-beam lithography technique [1].

In conclusion, low-resistance contacts are successfully formed for CNF interconnect devices using e- 
beam-deposited W after optimizing the GIS parameters in the SEM chamber, and the results are comparable to those for FIB-deposited W contacts [2] as well as for $\mathrm{Au} / \mathrm{Ti}$ contacts fabricated using e-beam lithography [1].

References

[1] L. Zhang, D. Austin, V. I. Merkulov, A. V. Meleshko, K. L. Klein, M. A. Guillorn, D. H. Lowndes and M. L. Simpson, Appl. Phys. Lett. 84, 3972 (2004).

[2] T. Saito, T. Yamada, D. Fabris, H. Kitsuki, P.Wilhite, M. Suzuki, and C.Y. Yang, Appl. Phys. Lett. 93, 102108 (2008).

[3] D.C. Joy and P. D. Rack, Microscopy and Microanalysis, NY, 11, 816 (2005).

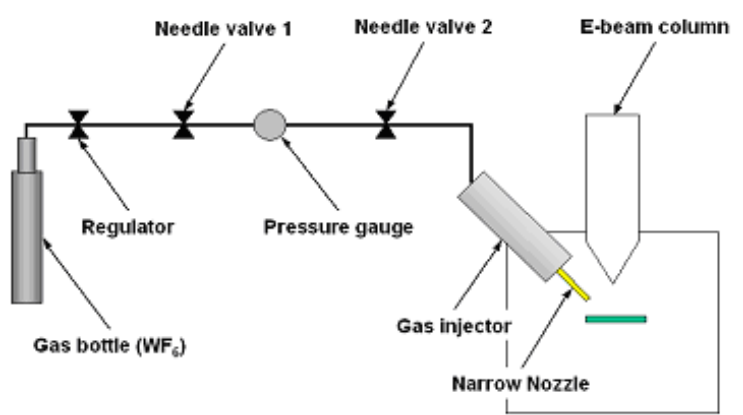

FIG. 1. Schematic of e-beam-induced deposition apparatus including a gas injection system (GIS).
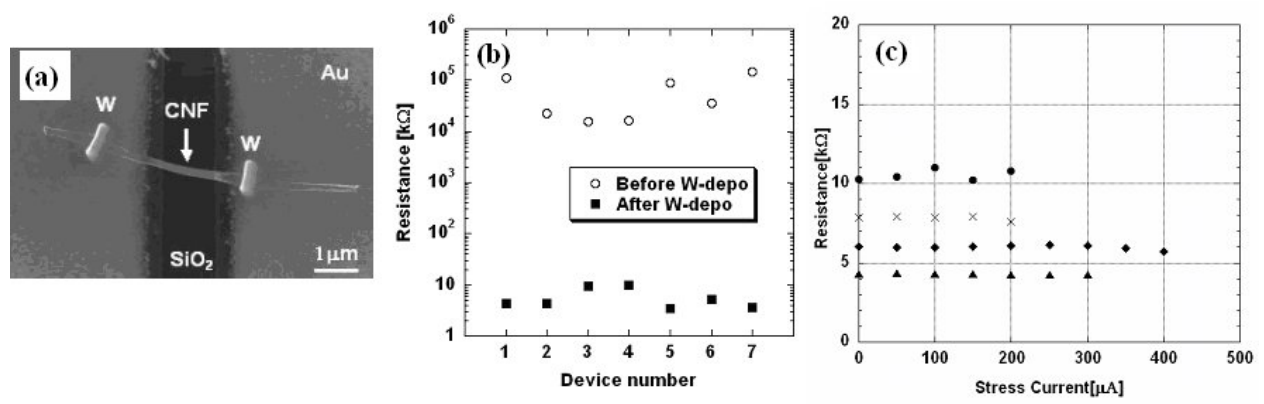

FIG. 2. (a) SEM image of CNF test device with W-deposited contacts. (b) Resistances of seven CNF test devices before and after W contact-deposition. (c) Resistances of four of the seven CNF test devices as a function of stress current.
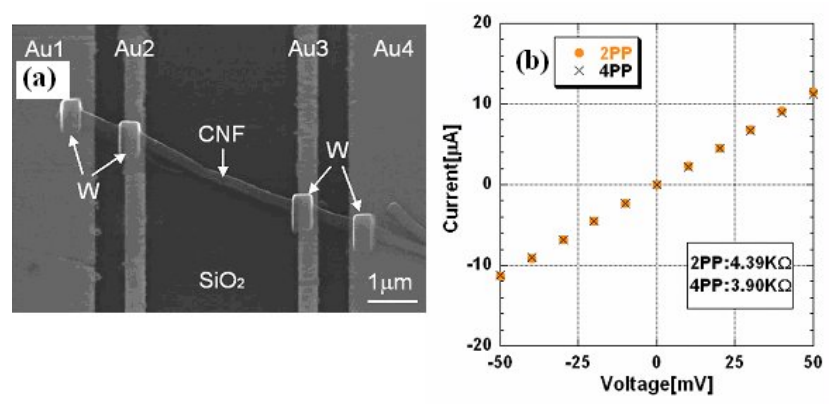

FIG. 3. SEM image (a) and $I-V$ characteristics (b) of CNF test device from two-point and four-point probe measurements. 